\title{
Study on the Prediction Methods of Aero Engine Fault Parameters Li Dewang ${ }^{a}$, Jiang Dongxiang ${ }^{\text {b }}$
}

(State Key Laboratory of Control and Simulation of Power System and Generation Equipments, De partment of Thermal Engineering, Tsinghua University, Beijing 100084, China.)

a Email: lidewangaiyu@126.com

${ }^{\text {b}}$ Email: jiangdx@mail.tsinghua.edu.cn

Keywords: aero-engine, characteristic parameters, prediction methods

\begin{abstract}
This paper uses the least squares method, BP neural network and gray GM(1,1) model for trend forecasts and predicting the remaining life of the aero-engine fault parameters. By comparing the results of the three methods, the least squares method and BP neural network method predicting results are more exact than the gray $\mathrm{GM}(1,1)$ model. The three methods are also used for predicting the actual data, and the result is close to the real.
\end{abstract}

\section{Introduction}

According to the statistics [1], though the proportion of the failure of aero engine causing plane to crash with the promotion of technology reduced in the domestic and foreign aircraft accidents, a certain proportion of the faults are left. Therefore, in order to protect the safety of people's lives and property safety of the country, Researching on the fault diagnosis forecast of aero engine is significant. The domestic researchers have made the trends and predict of the shaft wear and lubrication performance, the results show well. Abroad is mainly aimed at monitoring and research of engine health system, and the main engine company in the EHM project has made significant progress [2-4]. But on the whole, aero engine fault feature prediction and fault pre diagnosis do not develop well, so in order to fit the maintenance requirements, further studying on the prediction of fault feature parameters and the corresponding fault pre diagnosis is necessary.

Aero engine is the heart of aircraft and the complex system in the aircraft system, the main components of the engine work in high temperature and high pressure, high load demanding in long-term. Due to reasons such as fatigue or wear failure, which will affect the normal work of the engine [5]. Therefore, to ensure the engine safely operating, reflecting the state of the engine performance parameters plays an important role. The main parameters which can reflect the performance of the engine include pressure, temperature and speed. Trend prediction of these parameters will reflect the aero engine performance good recession, so the aero engine state can be judged, and the maintenance of aero engine fault can be effectively carried out.

\section{Engine simulation model}

As the absence of actual data, the mathematical model of aero engine is built to get the performance data. The aero engine is a very complex nonlinear system, so the simplified model can be achieved without concerning about the influence of combustion delay and viscous. Fig 1 shows the structure diagram of each part.

With the simulation of the engine model, the whole process data of the main parameters with fault could be got, then used to predict the fault parameters analysis. 


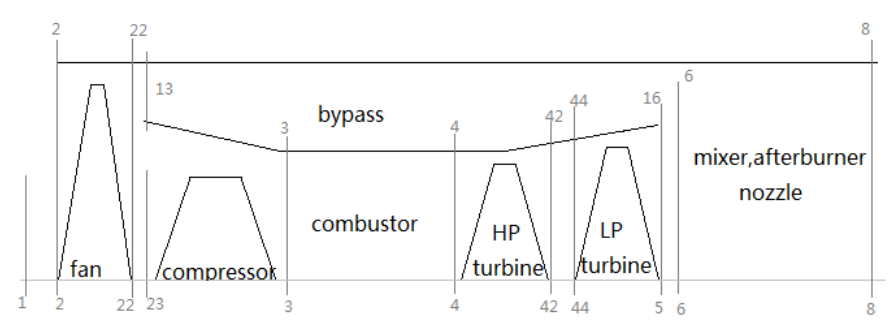

Fig 1 The Structure diagram of each part

\section{Study of prediction algorithm}

The least squares method. According to the definition of linear independence can be stated as follows the principle of least square method [6].

Supposing $\varphi_{0}(x), \varphi_{1}(x), \ldots, \varphi_{n-1}(x)$ are a continuous function of linear independence, defined in the interval $[a, b] . f(x)$ is a discrete function defined in the interval [a,b] with $n$ nodes as $\mathrm{a}<\mathrm{x}_{1}<\mathrm{x}_{2}<\ldots<\mathrm{x}_{\mathrm{n}}<\mathrm{b}$. the least squares method uses $\varphi_{0}(\mathrm{x}), \varphi_{1}(\mathrm{x}), \ldots, \varphi_{\mathrm{n}-1}(\mathrm{x})$ to make the Linear combination, then the combination function $F(x)=m_{0} \varphi_{0}(x)+m_{1} \varphi_{1}(x)+\ldots+m_{n-1} \varphi_{n-1}(x)$ is used to approach the function $f(x)$, which makes weighted sum of squares of $F(x)$ and $f(x)$ the smallest in all the combination function of $\varphi_{0}(x), \varphi_{1}(x), \ldots, \varphi_{n-1}(x)$. The square is:

$$
\sum_{i=1}^{n} \delta_{i}\left(f\left(x_{i}\right)-\sum_{j=0}^{n-1} m_{j} \varphi_{j}\left(x_{i}\right)\right)^{2}
$$

The weight $\delta_{\mathrm{i}}>0(1 \leq \mathrm{i} \leq \mathrm{n})$ is depended on the decision accuracy of measured data, with the different accuracy of classification, the weights are different, when the accuracy of classification is equal-precision, then $\delta_{\mathrm{i}}=1(1 \leq \mathrm{i} \leq \mathrm{n})$.

BP neural network. Multilayer feed forward neural network based on the structure of BP neural network algorithm is shown in Figure 2, The network is composed of three layers of network. The nodes in the same layer are without any coupling relationship. I.e. the output of each node only affects the output results of the next layer of nodes. The learning process of network is divided into forward and backward propagation, The characteristics of propagation of nodes is usually expressed as:

$$
f(x)=\frac{1}{1+e^{-b x}}, b>0
$$

The process of realization of the entire network is: the prepared data during the training phase is used to the input layer, hidden layer and output layer in turn, then comparing the output values and the expected values, if the error does not meet the requirements, continue training.

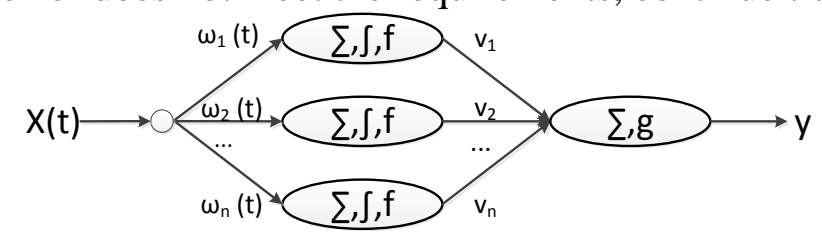

Fig 2 The structure of BP neural network model

Gray GM(1,1) model. The grey system is a system which contains unknown information of black also includes the known information of white. The main feature is modeling simply, requiring less sample size and solving prediction formula easily.

Initial data is $\mathrm{X}^{(0)}=\left\{\mathrm{x}^{(0)}(1), \mathrm{x}^{(0)}(2), \ldots \mathrm{x}^{(0)}(\mathrm{n})\right\}, \mathrm{x}^{(0)}(\mathrm{i})>0, \mathrm{i}=1,2, \ldots, \mathrm{n}$, calculating stepwise ratio $\sigma(\mathrm{i})=\mathrm{x}^{(0)}(\mathrm{i}-1) /$ $x^{(0)}(i), i=1,2, \ldots, n$, if the ratio is among the interval $\left(e^{-2 /(n+2)}, e^{2 /(n+2)}\right)$, then the data can be used directly in the gray $\mathrm{GM}(1,1)$ model for predicting, else calling for the pretreatment of the data, getting a new data series

The gray GM(1,1) model can be whitened to get the whitening equation, as: 


$$
\frac{d x^{(1)}}{d t}+a x^{(1)}=b
$$

$\mathrm{a}$ and $\mathrm{b}$ are the elements of the vector $\mathrm{A}$, as $\mathrm{A}=[\mathrm{a}, \mathrm{b}]^{\mathrm{T}}$. $\mathrm{a}$ and $\mathrm{b}$ can be solved by the least squares method. Then the response function of whitening equation can be solved, and discretized as:

$$
\widehat{x}^{(1)}(k)=\left(x^{(0)}(1)-\frac{b}{a}\right) e^{-a(k-1)}+\frac{b}{a}, k=2,3, \cdots, n .
$$

Then the predicting arrays are obtained as:

$$
\widehat{X}^{(0)}=\left\{\widehat{X}^{(0)}(1), \widehat{X}^{(0)}(2), \cdots, \widehat{X}^{(0)}(n)\right\}, k=1,2, \cdots, n \text {. }
$$

\section{Comparative analysis of algorithms}

Simulation data can be got by programming the three algorithms, then we can analyze the data and make the analysis of fault feature parameters trending and the remaining time, find out the pros and cons of the three algorithms.

Analysis of the characteristic parameters trending. This paper mainly uses simulation method to get the aero engine fault data, then the trend of these parameters changing can be learned through the different faults of engine performance. The main types of drop simulation performance are the fan efficiency fault, compressor efficiency fault, combustor efficiency fault and turbine efficiency fault. According to the actual measurement of the aero engine, this paper mainly analyzes the exhaust temperature of the simulation data.

Fig 3 shows the trend of exhaust temperature, when the combustion efficiency decreased.
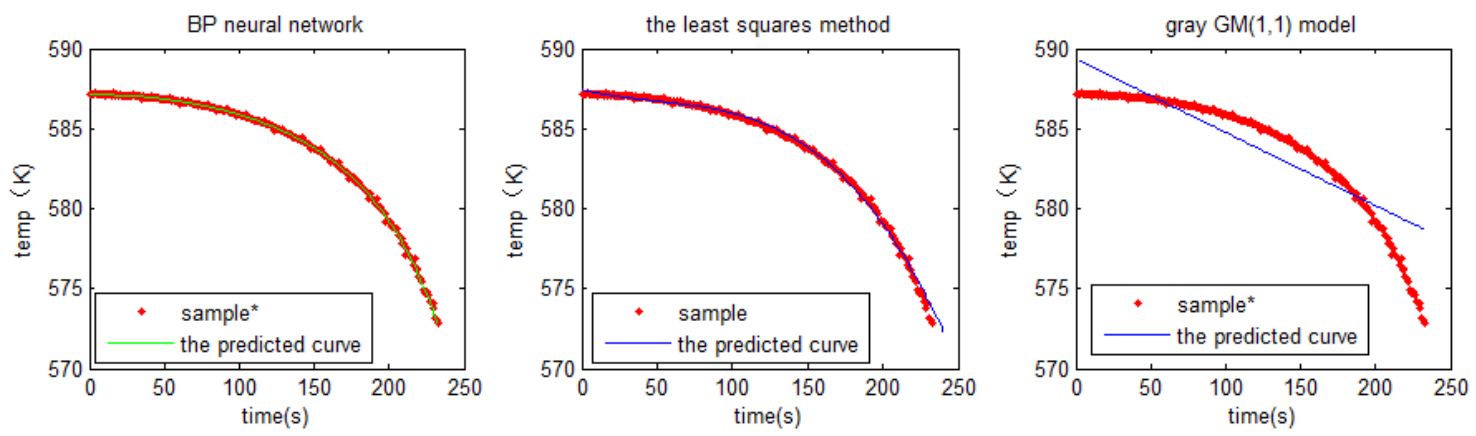

Fig 3 The trend of exhaust temperature when combustion efficiency faults

The prediction results are collected in the table 1, which can obviously makes out the comparison.

Table 1 Three different algorithms of predictive value and residual error

\begin{tabular}{c|c|c|c|c|c|c|c}
\hline time & $\begin{array}{c}\text { true value } \\
(\mathrm{K})\end{array}$ & $\begin{array}{c}\text { predictive } \\
\text { value 1 }(\mathrm{K})\end{array}$ & $\begin{array}{c}\text { predictive } \\
\text { value 2 }(\mathrm{K})\end{array}$ & $\begin{array}{c}\text { predictive } \\
\text { value 3 }(\mathrm{K})\end{array}$ & $\begin{array}{c}\text { error 1 } \\
(\mathrm{K})\end{array}$ & $\begin{array}{c}\text { error 2 } \\
(\mathrm{K})\end{array}$ & $\begin{array}{c}\text { error 3 } \\
(\mathrm{K})\end{array}$ \\
\hline 223 & 579.056 & 579.107 & 582.669 & 579.111 & 0.051 & 3.613 & 0.002 \\
224 & 578.866 & 578.927 & 582.631 & 578.933 & 0.061 & 3.765 & 0.134 \\
225 & 578.379 & 578.745 & 582.593 & 578.751 & 0.365 & 4.214 & 0.054 \\
226 & 578.216 & 578.559 & 582.555 & 578.567 & 0.342 & 4.339 & 0.066 \\
227 & 578.261 & 578.369 & 582.517 & 578.380 & 0.108 & 4.256 & 0.372 \\
228 & 578.076 & 578.177 & 582.479 & 578.190 & 0.101 & 4.403 & 0.351 \\
229 & 577.756 & 577.981 & 582.441 & 577.997 & 0.225 & 4.685 & 0.119 \\
230 & 577.674 & 577.782 & 582.403 & 577.801 & 0.107 & 4.729 & 0.114 \\
231 & 577.412 & 577.579 & 582.366 & 577.602 & 0.167 & 4.954 & 0.241 \\
232 & 577.661 & 577.372 & 582.328 & 577.399 & 0.288 & 4.653 & 0.127 \\
\hline
\end{tabular}

note: 1 is the least squares method, 2 is BP neural network, 3 is Gray GM(1,1) model

Figure 3 and Table 1 show that the gray $\operatorname{GM}(1,1)$ model result is bad, cannot fit the required for the trend analysis, but the least squares method and BP neural network do well, the errors are small.

As exponential equation is used in the gray $\operatorname{GM}(1,1)$ model, when the actual data does not fit the 
exponential law, the error will be large, and will be added up along with the amount of data. So the gray GM(1,1) model is bad for predicting the trend of parameters.

The least squares method uses the curve fitting method, as the fitting order is good, the prediction result will match the actual result, so this method can be used for trend analysis.

BP neural network depends on the notes number. Though the learning time is long, the predicting result is satisfied. Compared with the least squares method, the limitation of BP neural network is small, and less affected by the types of data. As data type in this paper is fixed, both the methods are good for predicting the trend.

Figure 4 shows predictions from a set of the literature data [7] obtained. This set of data is the deviation of the cruise state exhaust temperature value, the predicting result shows that all three methods are satisfied for predicting little data of actual situation.
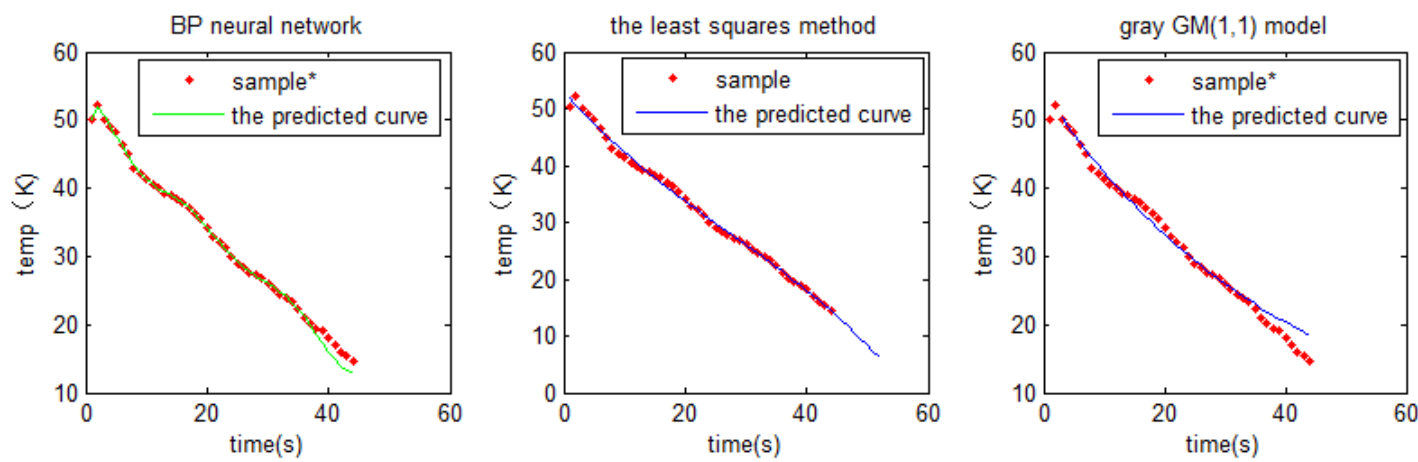

Fig 4 Trend analysis of the literature data

Analysis of effective remaining time. As BP neural network cannot get the forecasting function, the equation cannot be dealt to receive the time when the characteristic parameters reaching the critical value. So this paper uses the interpolation method, which can effectively reduce the calculation error, and obtain relatively satisfactory results. The method is as follows:

When the predicted curve through the critical value, the left and right integer time points of intersection are $t_{1}$ and $t_{2}$, corresponding to the actual curve values are $y_{1}$ and $y_{2}$, as the intervals are short, and $t_{1}, t_{2}$ are approximate among the whole predicting, so the prediction curve could be regarded as a straight line between $t_{1}$ and $t_{2}$, Then the value of the intersection can be got through linear interpolation, as:

$$
t_{\text {pred }}=\frac{t_{1}\left|y_{2}-y_{0}\right|+t_{2}\left|y_{1}-y_{0}\right|}{\left|y_{1}-y_{0}\right|+\left|y_{2}-y_{0}\right|} \text {. }
$$

The effective remaining time of the least squares method, BP neural network and gray GM(1,1) model can be solved through the above formula. Fig 5 shows that the effective remaining time of the exhaust temperature when the combustion efficiency dropped.
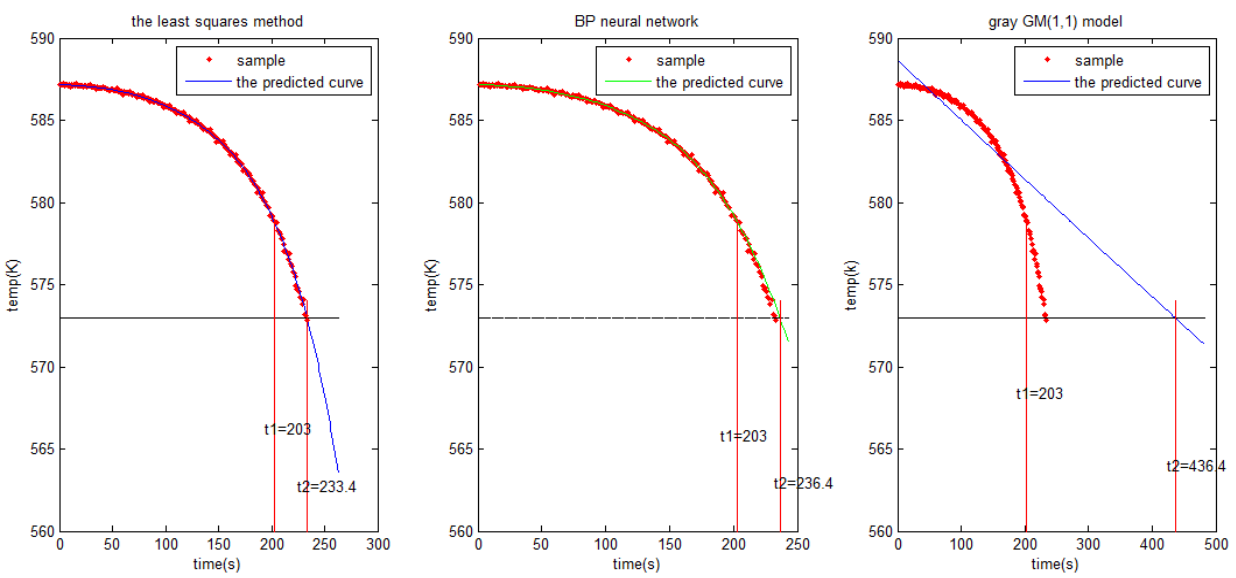

Fig 5 Prediction of the remainder of exhaust temperature when combustion efficiency faults

The first picture in Fig 5 shows that the difference between $t_{2}$ and $t_{1}$ can solve the remaining 
time when the exhaust temperature reaching the critical value, as $t_{1}$ is the starting predicting time, and $t_{2}$ is the reaching the critical value time, so the result is $\Delta t=t_{2}-t_{1}=30.4 s$. However, the predicting result depends on the Interval data points of the simulation model. Then the predicting time of BP neural network is $\Delta \mathrm{t}=\mathrm{t}_{2}-\mathrm{t}_{1}=33.4 \mathrm{~s}$, the gray $\mathrm{GM}(1,1)$ model is $\Delta \mathrm{t}=\mathrm{t}_{2}-\mathrm{t}_{1}=233.4 \mathrm{~s}$, the real remaining time is $28 \mathrm{~s}$.

Compared with the three methods, the least squares method is the best, BP neural network is a little bad, but gray GM(1,1) model cannot do the predicting, even worse it will give a wrong guidance.
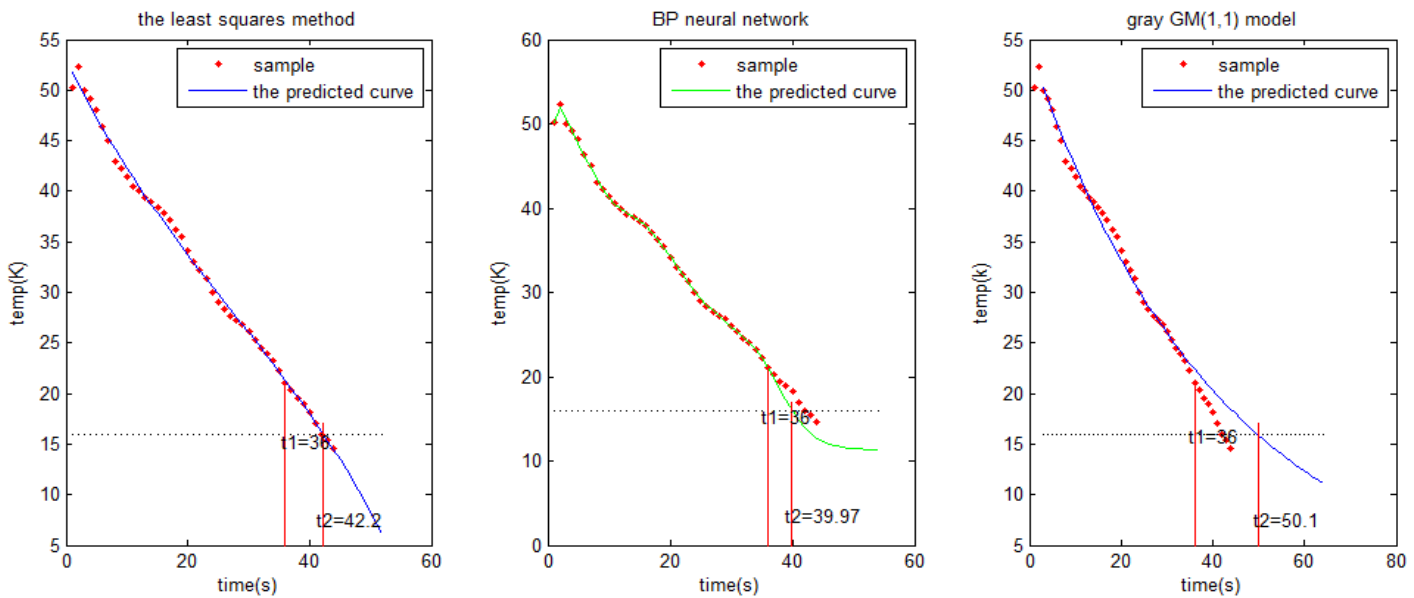

Fig 6 Predicting time of the literature data

Fig 6 shows the predicting time of the literature data, the remaining times are $t_{\text {pred } 1}=6.2 \mathrm{~s}$, $\mathrm{t}_{\text {pred } 2}=4.0 \mathrm{~s}, \mathrm{t}_{\text {pred } 3}=14.1 \mathrm{~s}$, the real time is $\mathrm{t}=6.0 \mathrm{~s}$, so the least squares method is the closest to the reality, but the three methods all can do the prediction.

\section{Conclusion}

This paper has finished the trend analysis of characteristic parameter and predicting of the remaining time. All the analysis depends on the exhaust temperature of the combustor efficiency fault. The trend analysis result shows that the gray $\mathrm{GM}(1,1)$ model cannot stress trend Change of parameters, the least squares method and BP neural network method are similar, can predict the trend of the parameters. The remaining time result shows that the gray $\operatorname{GM}(1,1)$ method predicts the time much longer than the real, cannot fit the practical requirements, otherwise, the least squares method does the best. According to the literature data, the least squares method is the best among the three methods, but BP neural network is close to the least squares method, the gray $\mathrm{GM}(1,1)$ method is the worst.

\section{Acknowledgements}

This work was supported by National Natural Science Foundation of China (Grant No. 60979014)

\section{References}

[1] Hu Haolei, Research on the prediction technology of engine removal in the condition based maintenance management of Aero-engine, Nanjing University of Aeronautics and Astronautics, Nanjing. (Chinese)

[2] Ashish Babbar, Estefan M. Ortiz, Advanced Diagnostics and Prognostics for Engine Health Monitoring, IEEEAC paper,2008.

[3] Kacprzynski G J, Roemer M J, Modgil G. Enhancement of physics of failure prognostic models with system level features, Proceedings of IEEE Aerospace Conference. 2002: 2919-2925. 
[4] David J. Bryg, George Mink, Link C. Jaw, Combining Lead Functions and Logistic Regression for Predicting Failures on An Aircraft engine, Proceedings of ASME Turbo Expo 2008: Power for Land, Sea and Air.

[5] Liu Zhiguo, Wang Jin, Forecasting Research on Aero-engine Rotate Speed Signal Based on ARMA Model, Aeronautical Computing Technique. July 2014,vol. 41: 85-88. (Chinese)

[6] Ding Gang, Xu Minqiang, Prediction of aero-engine exhaust gas temperature using process neural network, Journal of Aerospace Power. May 2009,vol. 24: 1035-1039. (Chinese)

[7] Chen Guo, Forecasting Engine Performance Trend by Using Structure Self-Adaptive Neural Network, Acta Aeronautica et Astronautica Sinica. May 2007,vol. 28: 535-539. (Chinese) 